\title{
Some nonexistence results for positive solutions of elliptic equations in unbounded domains
}

Lucio Damascelli and Francesca Gladiali

\begin{abstract}
We prove some Liouville type theorems for positive solutions of semilinear elliptic equations in the whole space $\mathbb{R}^{N}, N \geq 3$, and in the half space $\mathbb{R}_{+}^{N}$ with different boundary conditions, using the technique based on the Kelvin transform and the Alexandrov-Serrin method of moving hyperplanes. In particular we get new nonexistence results for elliptic problems in half spaces satisfying mixed (Dirichlet-Neumann) boundary conditions.
\end{abstract}

\section{Introduction and statement of the results}

In a famous paper [15] Gidas and Spruck proved, as a byproduct of much more general results, a general Liouville type theorem for subcritical elliptic equations, namely that there are not nontrivial $C^{2}$ solutions (without any condition at infinity) of the problem

$$
\begin{cases}-\Delta u=u^{p} & \text { in } \mathbb{R}^{N} \\ u \geq 0 & \text { in } \mathbb{R}^{N}\end{cases}
$$

if $N \geq 3$ and $1<p<\frac{N+2}{N-2}$.

As it is well known Liouville type theorems are important in many situations, for example in proving a priori estimates (see e.g. [16]).

Later Chen and $\mathrm{Li}$ [10] found a simple proof of the previous result based on the Kelvin transform and the Alexandrov-Serrin moving plane method,

2000 Mathematics Subject Classification: 35B05, 35B45, 35B50.

Keywords: Liouville theorems, Kelvin transform, maximum principle, moving plane. 
and the same technique also shows that if $p=\frac{N+2}{N-2}$ the only solutions to (1.1) are the well known solutions having the form

$$
u(x)=\frac{\left[N(N-2) \lambda^{2}\right]^{\frac{N-2}{4}}}{\left(\lambda^{2}+\left|x-x^{0}\right|^{2}\right)^{\frac{N-2}{2}}}
$$

for some $\lambda>0$ and $x^{0} \in \mathbb{R}^{N}$. This last result had been proved by Gidas, Ni and Nirenberg [14] for solutions with some decay at infinity and by Caffarelli, Gidas and Spruck [8] without any condition at infinity.

The Kelvin transform together with the moving plane method was exploited also by Gidas and Spruck in another paper [16] to prove that there are not nontrivial solutions of the corresponding Dirichlet problem in a half space, namely

$$
\begin{cases}-\Delta u=u^{p} & \text { in } \mathbb{R}_{+}^{N}=\left\{x=\left(x^{1}, \ldots, x^{N}\right): x^{N}>0\right\} \\ u \geq 0 & \text { in } \mathbb{R}_{+}^{N} \\ u=0 & \text { on } \partial \mathbb{R}_{+}^{N}\end{cases}
$$

if $N \geq 3$ and $1<p \leq \frac{N+2}{N-2}$. Using both type of Liouville theorems the authors prove some general a priori estimates for solutions of subcritical semilinear elliptic equations.

For what concerns the corresponding problem in a half space with mixed boundary conditions Berestycki, Grossi and Pacella [7] proved that there are not nontrivial solutions belonging to the space $D^{1,2}\left(\mathbb{R}_{+}^{N}\right)$ of the problem

$$
\begin{cases}-\Delta u=u^{p} & \text { in } \mathbb{R}_{+}^{N} \\ u \geq 0 & \text { in } \mathbb{R}_{+}^{N} \\ u=0 & \text { on } \Gamma_{0}=\left\{x=\left(x^{1}, \ldots, x^{N}\right): x^{N}=0, x^{1}>0\right\} \\ \frac{\partial u}{\partial x^{N}}=0 & \text { on } \Gamma_{1}=\left\{x=\left(x^{1}, \ldots, x^{N}\right): x^{N}=0, x^{1}<0\right\}\end{cases}
$$

where $N \geq 3, p=\frac{N+2}{N-2}$, using again the Kelvin transform and the moving plane method.

Since then the technique based on the Kelvin transformation and the moving plane method has been then used by many authors to prove Liouville type results for nonnegative solutions of different kind of elliptic problems, in $\mathbb{R}^{N}$ and for Dirichlet or nonlinear Neumann problems in half spaces (see e.g. [2], [9], [17], [20], [21]). 
In particular Chen and Lin [9] proved that if $f:[0, \infty) \rightarrow \mathbb{R}$ satisfies the following assumptions

(1) $f(s) \geq 0$

(2) $f$ is nondecreasing

(3) $f$ is locally Lipschitz

(4) $\frac{f(t)}{t^{N+2}}$ is nonincreasing in $(0,+\infty)$.

then there are no nontrivial solutions of the problem

$$
\begin{cases}-\Delta u=f(u) & \text { in } \mathbb{R}^{N} \\ u \geq 0 & \text { in } \mathbb{R}^{N}\end{cases}
$$

unless there exists $l \geq 0$ such that $f(t)=l t^{\frac{N+2}{N-2}}$ in the range of $u$. Condition (2) was then dropped by Bianchi [2] that also treated nonlinearity depending on $|x|$ and other problems.

Lou and Zhu [17] studied problems in half spaces with nonlinear Neumann boundary conditions and also considered the problem (1.4) when the nonlinearity has the opposite sign, namely $f(u)=-u^{p}$ and proved that there are no solutions for any $p>1$ using the same technique.

In all this papers the classical moving plane method based on pointwise estimate and the maximum principle was exploited. In particular maximum principles for singular solutions of differential inequalities (see [19]) were used to guarantee that positive solutions with a possible isolated singularity are bounded from below by a positive constant in punctured balls around the singularity, and this was the condition that allows to start the method. This is why the condition (1) appears in some of the previous papers and different kind of maximum principles are needed in treating negative superlinear nonlinearities. This is also the reason why a different technique, exploiting a Kelvin transform that maps the half space in a ball, is used in [16] for the Dirichlet problem in $\mathbb{R}_{+}^{N}$ : the (singular) solution cannot be greater than a positive constant in $\mathbb{R}_{+}^{N} \cap B(0, r) \backslash\{0\}$ because of the boundary condition.

Another technique used together with the moving plane method to prove symmetry results is based on integral inequality, as used by Terracini in two interesting papers [20], [21] where singular elliptic problems in $\mathbb{R}^{N}$ or in the half space with nonlinear Neumann data on the boundary are treated (see also [1] and [13] where this technique was used in studying the symmetry of positive solutions of elliptic problems on manifold and involving the $p$-Laplace operator). 
In this paper we get new nonexistence results for a general class of semilinear elliptic problems in half spaces with mixed (Dirichlet-Neumann) boundary conditions, i.e. the problem

$$
\begin{cases}-\Delta u=f(u) & \text { in } \mathbb{R}_{+}^{N} \\ u \geq 0 & \text { in } \mathbb{R}_{+}^{N} \\ u=0 & \text { on } \Gamma_{0}=\left\{x=\left(x^{1}, \ldots, x^{N}\right): x^{N}=0, x^{1}>0\right\} \\ \frac{\partial u}{\partial x^{N}}=0 & \text { on } \Gamma_{1}=\left\{x=\left(x^{1}, \ldots, x^{N}\right): x^{N}=0, x^{1}<0\right\}\end{cases}
$$

Here $N \geq 3$ and $f:[0,+\infty) \rightarrow \mathbb{R}$ is a continuous function.

Let us put $A=\mathbb{R}_{+}^{N} \cup \Gamma_{1}$ and $W=\left\{\varphi \in C_{c}^{1}\left(\overline{\mathbb{R}_{+}^{N}}\right): \operatorname{supp}(\varphi) \subseteq A\right\}$.

By a weak solution of (1.5) we mean a function $u \in W_{\text {loc }}^{1,2}\left(\mathbb{R}_{+}^{N}\right) \cap L_{\text {loc }}^{\infty}\left(\overline{\mathbb{R}_{+}^{N}}\right)$ (i.e. $u$ is bounded in $K \cap \mathbb{R}_{+}^{N}$ for every compact $K \subseteq \mathbb{R}^{N}$ ) such that $u=0$ on $\Gamma_{0}$ and

$$
\int_{\mathbb{R}_{+}^{N}} \nabla u \nabla \varphi=\int_{\mathbb{R}_{+}^{N}} f(u) \varphi
$$

for every $\varphi \in W$. Our main result is the following

Theorem 1.1 Let $u \in W_{\text {loc }}^{1,2}\left(\mathbb{R}_{+}^{N}\right) \cap C^{0}\left(\overline{\mathbb{R}_{+}^{N}}\right)$ be a weak solution of problem (1.5) in $\mathbb{R}_{+}^{N}$, where $N \geq 3$ and $f:[0, \infty) \rightarrow \mathbb{R}$ is a continuous function satisfying

i) $g(t)=\frac{f(t)}{t^{N+2}}$ is nonincreasing in $(0,+\infty)$.

ii) $\frac{f^{+}(t)}{t}$ is bounded for $t \rightarrow 0$.

Then $u$ depends only on $x^{1}$ and $x^{N}$ and is nonincreasing in the $x^{1}$-direction.

Moreover if $f$ satisfies $i)$, ii) and

iii) $f(s)>0$ for every $s>0, \quad \liminf _{t \rightarrow \infty} f(t)>0$

then $u \equiv 0$ is the only bounded solution of the problem (1.5).

As a particular case we get the nonexistence of nontrivial bounded solutions of the problem (1.3), for any $p$ with $1<p \leq \frac{N+2}{N-2}$ and without requiring any condition on the summability of the solution.

We use the technique based on the Kelvin transform together with the moving plane method, as it was done in previous papers dealing with problems in $\mathbb{R}^{N}$ or in $\mathbb{R}_{+}^{N}$ with Dirichlet conditions, but in developing the method we use integral inequalities, as used by Terracini in [20], [21], and more recently by one of the authors in [1]. The advantage of this approach is that 
the same method can be applied in different problems, in the whole space as well as in half spaces under different boundary conditions, and there is not any more the need for different maximum principles to start the method.

In fact we also get as a byproduct an unified proof of the known results for problems (1.4) and for the corresponding Dirichlet problem in the half space (see problem (1.6) that follows) which also gives some improvement of the known results. In particular we show that only condition (4) above on $f$ is needed to prove nonexistence for the problem (1.4) in the whole space, i.e. we prove the following

Theorem 1.2 Let $u \in W_{\text {loc }}^{1,2}\left(\mathbb{R}^{N}\right) \cap C^{0}\left(\mathbb{R}^{N}\right)$ be a (weak) solution of problem (1.4), where $N \geq 3$ and $f:[0, \infty) \rightarrow \mathbb{R}$ is a continuous function with the property

i) $g(t)=\frac{f(t)}{t^{N+2}}$ is nonincreasing in $(0,+\infty)$.

Then either $u \equiv c \in[0,+\infty)$ and $f(c)=0$, or there exist positive constants $k, h, l$ such that

$$
u(x)=\frac{k}{\left(h^{2}+\left|x-x_{0}\right|^{2}\right)^{\frac{N-2}{2}}}
$$

and $g(t)=l>0$, i.e. $f(t)=l t^{\frac{N+2}{N-2}}$.

Using the same technique one can consider solutions of the corresponding problem in the half space with Dirichlet boundary conditions, namely

$$
\begin{cases}-\Delta u=f(u) & \text { in } \mathbb{R}_{+}^{N}=\left\{x=\left(x^{1}, \ldots, x^{N}\right): x^{N}>0\right\} \\ u \geq 0 & \text { in } \mathbb{R}_{+}^{N} \\ u=0 & \text { on } \partial \mathbb{R}_{+}^{N}\end{cases}
$$

and prove the following analogous result.

Theorem 1.3 Let $u \in W_{\text {loc }}^{1,2}\left(\mathbb{R}_{+}^{N}\right) \cap C^{0}\left(\overline{\mathbb{R}_{+}^{N}}\right)$ be a (weak) solution of problem (1.6) in $\mathbb{R}_{+}^{N}$, where $N \geq 3$ and $f:[0, \infty) \rightarrow \mathbb{R}$ is a continuous function satisfying

i) $g(t)=\frac{f(t)}{t^{N+2}}$ is nonincreasing in $(0,+\infty)$.

ii) $\frac{f^{+}(t)}{t}$ is bounded for $t \rightarrow 0$.

Then $u$ depends only on $x^{N}$. Moreover if $f$ satisfies i), ii) and

iii) $f(s)>0$ for every $s>0, \quad \liminf _{t \rightarrow \infty} f(t)>0$

then problem (1.6) admits only the trivial solution $u \equiv 0$. 
Remark 1.1 Note that theorems 1.1-1.3 lead to a priori estimates in $L^{\infty}$ for problems in bounded domains with mixed boundary conditions. We refer to [16] for the corresponding analysis for Dirichlet BVP and to the recent paper [11] for a blow-up analysis for some mixed problems in bounded domains.

In this last paper, that we received after this paper was completed, the authors reach a priori bounds without using a general Liouville theorem as the previous one, but only proving that there are no solutions $u$ of the problem (1.5) in the case when $f(t)=t^{r}, 1<r<\frac{N+2}{N-2}$ having the property that $0 \leq u \leq 1=u((0,0, \ldots, 1))$.

The paper is organized as follows. In Section 2 we prove Theorems 1.2 and 1.3 as well as some related result, and show how the technique exploited permit to treat similarly different problems. We give a fairly detailed proof of Theorem 1.2, which is of course only a generalization of some known results, because the structure of the proof can be repeated with natural changes in the other results that we prove, in particular in the results on the mixed problem in a half space.

In Section 3 we focus on the mixed BVP in a half space and prove our main result, namely Theorem 1.1. We profit of the proofs given in Sect.2 to give a quick proof of the first part of the proof of Theorem 1.1. The proof of the monotonicity in the $x^{1}$-direction in the whole half space, which leads to nonexistence in some cases, is instead not straightforward and requires new ideas which are explained in detail.

We also state and prove a Liouville type result for nonnegative solutions belonging to a suitable function space.

Acknowledgements. We would like to thank Prof. Ireneo Peral for many interesting discussions and for showing us a preliminary version of the paper [11]. We also would like to thank Prof. Yuxin Ge for some useful suggestions about the manuscript.

\section{Proof of Theorems 1.2 and 1.3}

The proof of Theorem 1.2 will follow, as in [2] or directly, from the following

Proposition 2.1 Let $u$ and $f$ be as in Theorem 1.2 and suppose that $u$ is positive in $\mathbb{R}^{N}$. Let $v$ be the Kelvin transform of $u$ centered in a point $P$. Then $v$ is radial around some point $Q$.

Moreover if $g$ is not constant in $\left(0, \sup _{x \in \mathbb{R}^{N}} u(x)\right)$ then $Q=P$, i.e. $v$ is radial around the pole of the Kelvin transform.

Before giving the proof of Proposition 2.1 let us begin with some notations and comment. For simplicity of notations we will consider the Kelvin trans- 
form centered at the origin, namely

$$
v(x)=\frac{1}{|x|^{N-2}} u\left(\frac{x}{|x|^{2}}\right), \quad x \in \mathbb{R}^{N} \backslash\{0\}
$$

It satisfies (weakly) in $\mathbb{R}^{N} \backslash\{0\}$ the equation

$$
-\Delta v(x)=\frac{1}{|x|^{N+2}} f\left(|x|^{N-2} v(x)\right)
$$

which can also be written as

$$
-\Delta v(x)=g\left(|x|^{N-2} v(x)\right) v(x)^{\frac{N+2}{N-2}}
$$

where

$$
g(t)=\frac{f(t)}{t^{\frac{N+2}{N-2}}} .
$$

Moreover $v$ is continuous and strictly positive in $\mathbb{R}^{N} \backslash\{0\}$, with a possible singularity at the origin, and decays at infinity as $u(0)|x|^{2-N}$, so that $v \in$ $L^{2^{*}} \cap L^{\infty}\left(\mathbb{R}^{N} \backslash B_{r}(0)\right)$ for any $r>0$.

To prove that $v$ is radial we use the moving plane method and prove symmetry in every direction, and for simplicity of notations we choose the $x^{1}$-direction. We then set the usual notations of the moving plane method: if $\lambda \in \mathbb{R}$ we define $\Sigma_{\lambda}=\left[x^{1}>\lambda\right]=\left\{x=\left(x^{1}, \ldots, x^{N}\right): x^{1}>\lambda\right\}, T_{\lambda}=$ $\partial \Sigma_{\lambda}=\left[x^{1}=\lambda\right]=\left\{x=\left(x^{1}, \ldots, x^{N}\right): x^{1}=\lambda\right\}$, and for $x \in \Sigma_{\lambda}$ we denote by $x_{\lambda}=R_{\lambda}(x)=\left(2 \lambda-x^{1}, x^{2}, \ldots, x^{N}\right)$ the image of $x$ under the reflection through the hyperplane $T_{\lambda}$ and by $v_{\lambda}(x)=v\left(x_{\lambda}\right)$ the reflected function, which is singular in the point $P_{\lambda}=(2 \lambda, 0, \ldots, 0)$.

Finally let $\Lambda$ be the set of those $\lambda \in(0,+\infty)$ such that $v \leq v_{\mu}$ in $\Sigma_{\mu} \backslash P_{\mu}$ for every $\mu \in(\lambda,+\infty)$.

To prove that $v$ is symmetric with respect to some hyperplane $T_{\lambda}$ it suffices to prove that

Step 1: $\Lambda \neq \emptyset$

Putting $\lambda_{0}=\inf \Lambda$ then

Step 2: If $\lambda_{0}>0$ then $v \equiv v_{\lambda_{0}}$ in $\Sigma_{\lambda_{0}}$.

In fact if $\lambda_{0}=0$ then by continuity $v \leq v_{0}$ in $\Sigma_{0}$. Doing the same procedure with respect to the opposite direction it is easy to conclude that either $v \equiv v_{\lambda_{0}}$ in $\Sigma_{\lambda_{0}}$ for some $\lambda_{0}<0$ or $v \equiv v_{0}$ in $\Sigma_{0}$.

To handle the (eventual) singularity of $v_{\lambda}$ in $P_{\lambda}$, we first prove the following lemma, where we exploit a technique based on cut-off functions, as done by Terracini in [21]. 
Lemma 2.1 For any fixed $\lambda>0$ the functions $v$ and $\left(v-v_{\lambda}\right)^{+}$belong to $L^{2^{*}} \cap L^{\infty}\left(\Sigma_{\lambda}\right)$, the function $\left(v-v_{\lambda}\right)^{+}$belongs to $W^{1,2}\left(\Sigma_{\lambda}\right)$. Moreover if we put $A_{\lambda}=\left\{x \in \Sigma_{\lambda} \backslash P_{\lambda}: g\left(|x|^{N-2} v(x)\right)>0, v(x) \geq v_{\lambda}(x)\right\}$, there exists $C_{\lambda}>0$, nonincreasing in $\lambda$, such that

$$
\int_{\Sigma_{\lambda}}\left|\nabla\left(v-v_{\lambda}\right)^{+}\right|^{2} \leq C_{\lambda}\left(\int_{A_{\lambda}} \frac{1}{|x|^{2 N}}\right)^{\frac{2}{N}}\left(\int_{\Sigma_{\lambda}}\left|\nabla\left(v-v_{\lambda}\right)^{+}\right|^{2}\right)
$$

Proof. If $\lambda>0$ then there exists $r>0$ such that $\Sigma_{\lambda} \subseteq \mathbb{R}^{N} \backslash B_{r}(0)$, so that $v$ and $\left(v-v_{\lambda}\right)^{+} \leq v$ belong to $L^{2^{*}} \cap L^{\infty}\left(\Sigma_{\lambda}\right)$ and the function $\frac{1}{|x|^{2 N}}$ which appears in (2.1) is integrable in $\Sigma_{\lambda}$. The fact that $\left(v-v_{\lambda}\right)^{+}$belongs to $W^{1,2}\left(\Sigma_{\lambda}\right)$ will be a consequence of the estimate $(2.1)$.

For $\varepsilon>0$ small let $\eta=\eta_{\varepsilon} \in C_{c}^{1}\left(\mathbb{R}^{N}\right)$ be a cut-off such that $0 \leq \eta \leq 1$, $\eta(x)=1$ if $2 \varepsilon \leq\left|x-P_{\lambda}\right| \leq \frac{1}{\varepsilon}, \eta(x)=0$ if $\left|x-P_{\lambda}\right|<\varepsilon$ or $\left|x-P_{\lambda}\right|>\frac{2}{\varepsilon}$, $|\nabla \eta| \leq \frac{2}{\varepsilon}$ if $\varepsilon<\left|x-P_{\lambda}\right|<2 \varepsilon,|\nabla \eta| \leq 2 \varepsilon$ if $\frac{1}{\varepsilon}<\left|x-P_{\lambda}\right|<\frac{2}{\varepsilon}$.

We then test the equations

$$
-\Delta v(x)=g\left(|x|^{N-2} v(x)\right) v(x)^{\frac{N+2}{N-2}}, \quad-\Delta v_{\lambda}(x)=g\left(\left|x_{\lambda}\right|^{N-2} v\left(x_{\lambda}\right)\right) v_{\lambda}(x)^{\frac{N+2}{N-2}}
$$

in $\Sigma_{\lambda} \backslash P_{\lambda}$ with the function $\varphi=\varphi_{\varepsilon}=\eta_{\varepsilon}^{2}\left(v-v_{\lambda}\right)^{+}$, to obtain estimates for the function $\psi=\psi_{\varepsilon}=\eta_{\varepsilon}\left(v-v_{\lambda}\right)^{+}$.

Since $|\nabla \psi|^{2}=\nabla\left(v-v_{\lambda}\right) \cdot \nabla \varphi+\left[\left(v-v_{\lambda}\right)^{+}\right]^{2}|\nabla \eta|^{2}$ it follows, subtracting the equations, that

$$
\begin{array}{r}
\int_{\Sigma_{\lambda} \cap\left[2 \varepsilon \leq\left|x-P_{\lambda}\right| \leq \frac{1}{\varepsilon}\right]}\left|\nabla\left(v-v_{\lambda}\right)^{+}\right|^{2} \leq \int_{\Sigma_{\lambda}}|\nabla \psi|^{2}=\int_{\Sigma_{\lambda}} \nabla\left(v-v_{\lambda}\right) \cdot \nabla \varphi+I_{\varepsilon} \\
=\int_{\Sigma_{\lambda}}\left[g\left(|x|^{N-2} v(x)\right) v^{p}-g\left(\left|x_{\lambda}\right|^{N-2} v\left(x_{\lambda}\right)\right) v_{\lambda}^{p}\right] \varphi+I_{\varepsilon}
\end{array}
$$

where $p=\frac{N+2}{N-2}, \quad I_{\varepsilon}=\int_{\Sigma_{\lambda}}\left[\left(v-v_{\lambda}\right)^{+}\right]^{2}\left|\nabla \eta_{\varepsilon}\right|^{2}$.

Since $g$ is nonincreasing, $|x| \geq\left|x_{\lambda}\right|$ and $v(x) \geq v\left(x_{\lambda}\right)$ in the set where $\varphi>0$, we have that $-g\left(\left|x_{\lambda}\right|^{N-2} v\left(x_{\lambda}\right)\right) \leq-g\left(|x|^{N-2} v(x)\right)$ there, so that

$$
\begin{aligned}
\int_{\Sigma_{\lambda} \cap\left[2 \varepsilon \leq\left|x-P_{\lambda}\right| \leq \frac{1}{\varepsilon}\right]} \mid \nabla & \left.\left(v-v_{\lambda}\right)^{+}\right|^{2} \leq \int_{\Sigma_{\lambda}}\left[g\left(|x|^{N-2} v(x)\right)\right]\left(v^{p}-v_{\lambda}^{p}\right) \varphi+I_{\varepsilon} \\
& \leq \int_{\Sigma_{\lambda}}\left[g^{+}\left(|x|^{N-2} v(x)\right)\right]\left(v^{p}-v_{\lambda}^{p}\right) \varphi+I_{\varepsilon} \\
& =\int_{A_{\lambda}}\left[g^{+}\left(|x|^{N-2} v(x)\right)\right]\left(v^{p}-v_{\lambda}^{p}\right) \varphi+I_{\varepsilon}
\end{aligned}
$$


Moreover, since $u$ is positive and locally bounded, there exist $0<a=a_{\lambda}<$ $b=b_{\lambda}<+\infty$ such that

$$
a<|x|^{N-2} v(x)=u\left(\frac{x}{|x|^{2}}\right)<b \quad \forall x \in \Sigma_{\lambda} \subseteq \mathbb{R}^{N} \backslash B_{r}(0),
$$

so that $0 \leq g^{+}\left(|x|^{N-2} v(x)\right) \leq g^{+}\left(a_{\lambda}\right)=: C_{\lambda}$.

Finally if $0 \leq v_{\lambda} \leq v$ we have that

$$
v^{\frac{N+2}{N-2}}-v_{\lambda}^{\frac{N+2}{N-2}} \leq \frac{N+2}{N-2} v^{\frac{4}{N-2}}\left(v-v_{\lambda}\right) \leq C_{\lambda} \frac{1}{|x|^{4}}\left(v-v_{\lambda}\right),
$$

as $v \in L^{\infty}\left(\Sigma_{\lambda}\right), \lambda>0$, and decays at infinity as $\frac{1}{|x|^{N-2}}$.

From the previous estimate we then get, using Hölder's inequality,

$$
\begin{aligned}
\int_{\Sigma_{\lambda} \cap\left[2 \varepsilon \leq\left|x-P_{\lambda}\right| \leq \frac{1}{\varepsilon}\right]}\left|\nabla\left(v-v_{\lambda}\right)^{+}\right|^{2} \leq \int_{A_{\lambda}}\left[g^{+}\left(|x|^{N-2} v(x)\right)\right]\left(v^{p}-v_{\lambda}^{p}\right) \varphi+I_{\varepsilon} \\
\leq C_{\lambda} \int_{A_{\lambda}} \frac{1}{|x|^{4}} \eta^{2}\left[\left(v-v_{\lambda}\right)^{+}\right]^{2}+I_{\varepsilon} \\
\leq C_{\lambda}\left(\int_{A_{\lambda}} \frac{1}{|x|^{2 N}}\right)^{\frac{2}{N}}\left(\int_{\Sigma_{\lambda}} \eta^{2^{*}}\left[\left(v-v_{\lambda}\right)^{+}\right]^{2^{*}}\right)^{\frac{2}{2^{*}}}+I_{\varepsilon}
\end{aligned}
$$

We observe now that the term $I_{\varepsilon}$ tends to zero as $\varepsilon \rightarrow 0$.

In fact, if $B_{\varepsilon}=\left\{x \in \Sigma_{\lambda}: \varepsilon<\left|x-P_{\lambda}\right|<2 \varepsilon \mid\right.$ or $\left.\frac{1}{\varepsilon}<\left|x-P_{\lambda}\right|<\frac{2}{\varepsilon} \mid\right\}$, observe that $B_{\varepsilon} \rightarrow \emptyset$ as $\varepsilon \rightarrow 0$ and $\left(v-v_{\lambda}\right)^{+} \leq v \in L^{2^{*}}{ }^{\varepsilon}\left(\Sigma_{\lambda}\right)$.

Moreover

$$
\left|\nabla \eta_{\varepsilon}\right|^{N}\left|B_{\varepsilon}\right| \leq C\left(\frac{1}{\varepsilon^{N}} \varepsilon^{N}+\varepsilon^{N} \frac{1}{\varepsilon^{N}}\right)=C,
$$

so we get

$$
I_{\varepsilon} \leq \int_{B_{\varepsilon}}\left(\left[\left(v-v_{\lambda}\right)^{+}\right]^{2^{*}}\right)^{\frac{2}{2^{*}}}\left(\int_{\Sigma_{\lambda}}|\nabla \eta|^{N}\right)^{\frac{2}{N}} \leq C \int_{B_{\varepsilon}}\left(\left[\left(v-v_{\lambda}\right)^{+}\right]^{2^{*}}\right)^{\frac{2}{2^{*}}} \rightarrow 0
$$

as $\varepsilon \rightarrow 0$ because $\left(v-v_{\lambda}\right)^{+} \in L^{2^{*}}\left(\Sigma_{\lambda}\right)$.

Letting now $\varepsilon$ tend to zero and using monotone and dominated convergence and Sobolev's inequality, we get

$$
\begin{aligned}
\int_{\Sigma_{\lambda}}\left|\nabla\left(v-v_{\lambda}\right)^{+}\right|^{2} & \leq C_{\lambda}\left(\int_{A_{\lambda}} \frac{1}{|x|^{2 N}}\right)^{\frac{2}{N}}\left(\int_{\Sigma_{\lambda}}\left[\left(v-v_{\lambda}\right)^{+}\right]^{2^{*}}\right)^{\frac{2}{2^{*}}} \\
& \leq C_{\lambda}\left(\int_{A_{\lambda}} \frac{1}{|x|^{2 N}}\right)^{\frac{2}{N}} \int_{\Sigma_{\lambda}}\left|\nabla\left(v-v_{\lambda}\right)^{+}\right|^{2}
\end{aligned}
$$


Proof of Proposition 2.1 From the estimate 2.1 we deduce immediately that if $\lambda>0$ and $C_{\lambda}\left(\int_{A_{\lambda}} \frac{1}{|x|^{2 N}}\right)^{\frac{2}{N}}<1$ then $\int_{\Sigma_{\lambda}}\left|\nabla\left(v-v_{\lambda}\right)^{+}\right|^{2}=0$, so that $v \leq v_{\lambda}$ in $\Sigma_{\lambda}$. This allows to prove the two main steps of the moving plane method rather easily.

Step 1. Since $\frac{1}{|x|^{2 N}} \in L^{1}\left(\Sigma_{\lambda_{1}}\right)$ for every $\lambda_{1}>0$ and $\Sigma_{\lambda} \rightarrow \emptyset$ as $\lambda \rightarrow+\infty$, it follows that

$$
\int_{A_{\lambda}} \frac{1}{|x|^{2 N}} \leq \int_{\Sigma_{\lambda}} \frac{1}{|x|^{2 N}} \rightarrow 0 \quad \text { as } \quad \lambda \rightarrow \infty
$$

so that $C_{\lambda}\left(\int_{A_{\lambda}} \frac{1}{|x|^{2 N}}\right)^{\frac{2}{N}}<1$ for every $\lambda$ in some interval $\left(\lambda_{0},+\infty\right)$.

Step 2. Let $\lambda_{0}=\inf \Lambda$ be positive and suppose by contradiction that $v$ does not coincide with its reflection $v_{\lambda_{0}}$ in $\Sigma_{\lambda_{0}}$. Then we claim that

$$
v<v_{\lambda_{0}} \text { in the set } D_{\lambda_{0}}=\left\{x \in \Sigma_{\lambda_{0}} \backslash P_{\lambda_{0}}: g\left(|x|^{N-2} v(x)\right)>0\right\} \supset A_{\lambda_{0}} .
$$

This is not immediate, since $f$ is not supposed to be Lipschitz, but it can be readily verified as follows.

We know that $v \leq v_{\lambda_{0}}$ in $\Sigma_{\lambda_{0}} \backslash P_{\lambda_{0}}$ by continuity, and $|x|>\left|x_{\lambda_{0}}\right|$ in $\Sigma_{\lambda_{0}}$ because $\lambda_{0}>0$. Let us put $O=\left\{x \in D_{\lambda_{0}}:|x|^{N-2} v>\left|x_{\lambda_{0}}\right|^{N-2} v_{\lambda_{0}}\right\}$.

If $x \in D_{\lambda_{0}} \backslash O$ then $|x|^{N-2} v(x) \leq\left|x_{\lambda_{0}}\right|^{N-2} v_{\lambda_{0}}(x)$ so that

$$
v(x) \leq\left(\frac{\left|x_{\lambda_{0}}\right|}{|x|}\right)^{N-2} v_{\lambda_{0}}(x)<v_{\lambda_{0}}(x) .
$$

From this we get that $v<v_{\lambda_{0}}$ in $D_{\lambda_{0}} \backslash O$.

In the open set $O$ instead, since $g$ is positive and nonincreasing in $D_{\lambda_{0}}$ and $v \leq v_{\lambda_{0}}$ in $\Sigma_{\lambda_{0}}$, we have that

$$
-\Delta v(x)=g\left(|x|^{N-2} v(x)\right) v(x)^{\frac{N+2}{N-2}} \leq g\left(\left|x_{\lambda_{0}}\right|^{N-2} v\left(x_{\lambda_{0}}\right)\right) v_{\lambda_{0}}(x)^{\frac{N+2}{N-2}}=-\Delta v_{\lambda_{0}} .
$$

Note that the possibility that $v \equiv v_{\lambda_{0}}$ in a connected component $C$ of $O$ can happen only if $g=c$ in $C$ with the constant $c>0$ since $g\left(|x|^{N-2} v(x)\right)>0$ in $O$. It is easy to see then that $C=O=\Sigma_{\lambda_{0}} \backslash P_{\lambda_{0}}$, which is not possible since we are supposing that $v$ does not coincide with its reflection $v_{\lambda_{0}}$ in $\Sigma_{\lambda_{0}}$. So by the classical maximum principle we have that $v<v_{\lambda_{0}}$ in $O$, and since we already know that $v<v_{\lambda_{0}}$ in $D_{\lambda_{0}} \backslash O$ the claim is true.

This kind of strong comparison principle implies that the function $\frac{\chi_{A_{\lambda}}}{|x|^{2 N}}$, where $\chi_{S}$ stands for the characteristic function of the set $S$, converges pointwisely to zero as $\lambda \rightarrow \lambda_{0}$ in $\mathbb{R}^{N} \backslash\left(T_{\lambda_{0}} \cup\left\{P_{\lambda_{0}}\right\}\right)$ and hence almost everywhere.

If $0<\lambda_{0}-\delta<\lambda_{0}$ then $\frac{1}{|x|^{2 N}} \chi_{A_{\lambda}} \leq \frac{1}{|x|^{2 N}} \chi_{\Sigma_{\lambda_{0}-\delta}} \in L^{1}$, and by dominated convergence $\int_{A_{\lambda}} \frac{1}{|x|^{2 N}} \rightarrow 0$ as $\lambda \rightarrow \lambda_{0}$, so that $C_{\lambda}\left(\int_{A_{\lambda}} \frac{1}{|x|^{2 N}}\right)^{\frac{2}{N}}<1$ for $\lambda$ in some interval $\left(\lambda_{0}-\delta, \lambda_{0}\right)$. 
As before this implies that $v \leq v_{\lambda}$ in $\Sigma_{\lambda}$ for $\lambda<\lambda_{0}$ and close to $\lambda_{0}$, contradicting the inf properties of $\lambda_{0}$.

To prove the last assertion suppose that $\lambda_{0}>0$, so that $v \equiv v_{\lambda_{0}}$ as we just proved. This implies that $v$ is regular at the origin, i.e. $u$ is regular at infinity. For any $x \in \Sigma_{\lambda_{0}}$ we have $|x|>\left|x_{\lambda_{0}}\right|$ and $-\Delta v(x)=-\Delta v_{\lambda_{0}}(x)$, which implies that $g\left(|x|^{N-2} v(x)\right)=g\left(\left|x_{\lambda_{0}}\right|^{N-2} v\left(x_{\lambda_{0}}\right)\right)$ because $v \equiv v_{\lambda_{0}}$. Since $g$ is nonincreasing this implies that $g(t)$ is constant in a left neighborhood of $t$ for any $t$ of the form

$$
t=|x|^{N-2} v(x)=u\left(\frac{x}{|x|^{2}}\right), \quad x^{1}>\lambda_{0} .
$$

Analogously $g$ is constant in any right neighborhood of any $t=u\left(\frac{x}{|x|^{2}}\right)$, $x^{1}<\lambda_{0}$, in particular for any $t$ close to 0 , since $u$ tends to 0 at infinity. It is easy then to conclude that if $\lambda_{0}>0$ then $g$ is constant in $u\left(\mathbb{R}^{N}\right)$.

Proof of Theorem 1.2 First of all if (i) holds then either $u \equiv 0$ or $u$ is positive in $\mathbb{R}^{N}$. In fact if $u_{0}>0$ in the open set $O$ where $0 \leq u<u_{0}$ we have that

$$
-\Delta u=f(u)=g(u) u^{\frac{N+2}{N-2}} \geq g\left(u_{0}\right) u^{\frac{N+2}{N-2}}
$$

so that $-\Delta u+c u^{\frac{N+2}{N-2}} \geq 0$, which guarantees the validity of the strong maximum principle (see [22] and [18]).

If $u \equiv 0$ the theorem is proved, otherwise $u>0$ and by Proposition 2.1 the Kelvin transform $v$ of $u$ centered in any point $P$ is radially symmetric around some point $Q$. Moreover if $g$ is not constant on the values of $u$ then $Q=P$, which implies that also $u$ is radially symmetric around $P$. Since $P$ is arbitrary it follows that $u$ is constant.

If instead $g$ is constant then $f(t)=l t^{\frac{N+2}{N-2}}$ for every $t \in u\left(\mathbb{R}^{N}\right)$ and some $l \in \mathbb{R}$. Then either for every choice of the pole we have $\lambda_{0}=0$, in which case $u$ is constant, as we just proved, or there exists some pole $P$ for which $\lambda_{0}>0$. In this case $v \equiv v_{\lambda_{0}}$ in $\Sigma_{\lambda_{0}}$, so that 0 is not a singular point and $u$ is regular at infinity, i.e. decays at infinity as $\frac{1}{|x|^{N-2}}$.

Then necessarily $l>0$ (otherwise $u$ would be subharmonic with the maximum at some point) and by classical results (see [14], [8]) $u$ has the form indicated by the theorem.

Remark 2.1 Let us observe that once Proposition 2.1 is proved Theorem 1.2 follows immediately from the beautiful geometrical arguments of Bianchi (see Lemma 7 in [2]). In other situations however it is necessary to discuss separately the cases when $g$ is constant, as in the case of some problems in half spaces. 
As it is usual when proving nonexistence results with the aid of the Kelvin transformations and the moving plane method, the same technique can be exploited to prove symmetry results for singular solutions, as well as symmetry or nonexistence results for problems similar to (1.4) where the nonlinearity depends on $r=|x|$. As an example the following result can be proved by the same techniques. We refer to [2] and the references therein for other results of this type.

Theorem 2.1 Let $u \in W_{\text {loc }}^{1,2}\left(\mathbb{R}^{N} \backslash\{0\}\right) \cap C^{0}\left(\mathbb{R}^{N} \backslash\{0\}\right)$ be a (weak) solution of problem (1.4) in $\mathbb{R}^{N} \backslash\{0\}$, where $N \geq 3, f:[0, \infty) \rightarrow \mathbb{R}$ is as in Theorem 1.2 and 0 is a non removable singularity. Then $u$ is radial around the origin.

Proof. Let 0 be a non removable singularity of $u$ and let us consider the Kelvin transform $v$ of $u$ centered in a point $P=\left(P^{1}, \ldots, P^{N}\right)$ different from 0 but with $P^{1}=0$. Then $v$ is singular in two points which belong to the same central hyperplane $T_{0}=\left[x^{1}=0\right]$. Then a slight modification of Proposition 1 (2 singularities instead of 1 but both on the same central hyperplane) allows to conclude that $v$ is symmetric w.r.t. some hyperplane $T_{\lambda_{0}}=\left[x^{1}=\lambda_{0}\right]$, and since at least one singularity is non removable, necessarily $\lambda_{0}=0$.

So $v$ is symmetric w.r.t. to $T_{0}=\left[x^{1}=0\right]$, which implies that $u$ is also symmetric w.r.t. $T_{0}$. (The only difference with Theorem 1.2 is that now the symmetry of the Kelvin transform can be proved only if the pole is choosen in a particular way and cannot be arbitrary).

Repeating the procedure with respect to the other directions we conclude that $u$ is radially symmetric around the origin, i.e. the essential singularity point.

Let us now consider the case of the Dirichlet problem in a half space. We begin by observing that Theorem 1.3 deals with solutions that are not necessarily bounded, but for bounded solutions of (1.6) Theorem 1.2 and some known results by Dancer and Berestycki, Caffarelli and Nirenberg imply a general nonexistence result which we now describe.

Dancer [12] proved that if $f:[0, \infty) \rightarrow \mathbb{R}$ is a $C^{1}$ function satisfying $f(0)=0, f^{\prime}(0) \geq 0$, then any bounded solution $u$ of (1.6) is monotone increasing in the $x^{N}$-direction and the function $z\left(x^{1}, \ldots, x^{N-1}\right)=$ $\lim _{x^{N} \rightarrow \infty} u\left(x^{1}, \ldots, x^{N}\right)$ satisfies the same equation in $\mathbb{R}^{N-1}$.

Later Berestycki, Caffarelli and Nirenberg proved the monotonicity in the $x_{N}$-direction of any bounded solution under the hypothesis that $f$ is locally Lipschitz continuous in $[0, \infty)$ and $f(0) \geq 0$ if $N>2$ (see [6]). They actually consider solutions not necessarily bounded by supposing $f$ globally Lipschitz continuous and prove many other results on qualitative properties of positive solutions to elliptic problems in several unbounded domains in the series of papers [3]-[6]. 
A consequence of these results and Theorem 1.2 is the following

Corollary 2.1 Suppose that $N \geq 3, f:[0, \infty) \rightarrow \mathbb{R}$ is a locally Lipschitz function with $f(0) \geq 0$, and $u$ is a bounded solution of (1.6). Then necessarily $u \equiv 0$ if either $u$ tends to zero at infinity or $f$ satisfies

i) $f(s)>0$ if $s>0$;

ii) if $N>3, g(t)=\frac{f(t)}{t^{N+3}}$ is nonincreasing in $(0,+\infty)$ and non constant in any interval.

Let us only observe that if $N=3$ the results follows from the fact that the function $z\left(x^{1}, x^{2}\right)=\lim _{x^{3} \rightarrow \infty} u\left(x^{1}, x^{2}, x^{3}\right)$ is superharmonic and bounded in $\mathbb{R}^{2}$, so that it must be constant and by the positivity hypothesis on $f$ it must vanish. If instead $N>3$, the function $z\left(x^{1}, \ldots, x^{N-1}\right)=$ $\lim _{x^{N} \rightarrow \infty} u\left(x^{1}, \ldots, x^{N}\right)$ satisfy the same problem in $R^{N-1}$, so it must vanish by Theorem 1.2 , and this implies that $u$ vanishes in $\mathbb{R}_{+}^{N}$ because of the monotonicity in the $x^{N}$-direction.

Let us now prove Theorem 1.3.

Proof of Theorem 1.3. The proof is similar to that of Theorem 1.2. We consider the Kelvin transform $v$ of $u$ centered at a generic point $P \in \partial \mathbb{R}_{+}^{N}$, i.e. with $P^{N}=0$, and prove that it is symmetric w.r.t. some hyperplane $T_{\lambda_{0}}=\left[x^{1}=\lambda_{0}\right], \lambda_{0} \geq 0$, with $\lambda_{0}=0$ if $g$ is not constant on the values of $u$. If instead $g$ is constant then $f(t)=l t^{\frac{N+2}{N-2}}$ for some $l \geq 0$, but the case $\lambda_{0}>0$ cannot occur, unless $u$ vanishes identically, since in this case $v$ is regular in 0 , so $u$ is bounded and tends to zero at infinity. But then by the preceeding corollary $u$ vanishes identically.

Then we repeat the procedure with respect to all the directions orthogonal to the $x^{N}$-direction.

The proof of the symmetry of the Kelvin transforms of $u$ is essentially the same, with the test function and the solutions considered in spaces of functions defined in the half space of course, but with a main difference which is the following. In the case of the whole space the solution $u$ is positive in compact sets, so that in the proof of Lemma 2.1 we deduced that if $\lambda>0$ then for any $x \in \Sigma_{\lambda}$ the value $|x|^{N-2} v(x)=u\left(\frac{x}{|x|^{2}}\right)$ belongs to some interval $(a, b)$ for some $a, b$ depending on $\lambda, 0<a<b<\infty$.

In the present case instead $u\left(\frac{x}{|x|^{2}}\right)$ approaches 0 in compact sets, because of the boundary conditions, and this is the reason for the other condition ii).

This condition guarantees that $g^{+}(t) t^{\frac{4}{N-2}}=\frac{f^{+}(t)}{t}$ is bounded in any interval $(0, b)$ and allows to get the main estimate used in Lemma 2.1, namely if $|x|^{N-2} v(x)=u\left(\frac{x}{|x|^{2}}\right)$ is bounded from above, which is the case if $\lambda>0$ and 
$x \in \Sigma_{\lambda}$, then

$$
g^{+}\left(|x|^{N-2} v\right) v^{\frac{4}{N-2}}=\frac{g^{+}\left(|x|^{N-2} v\right)\left(|x|^{N-2} v\right)^{\frac{4}{N-2}}}{|x|^{4}} \leq C \frac{1}{|x|^{4}}
$$

Repeating the same prove we get that if $v$ is the Kelvin transform of $u$ centered at $P \in \partial \mathbb{R}_{+}^{N}$, then $v\left(., x^{N}\right)$ is radial around $P$, which implies that $u$ has the same property and, since $P$ is arbitrary, $u$ depends only on the $x^{N}$-variable.

If we have in addition the hypothesis iii) it is easy to prove that necessarily $u \equiv 0$ generalizing as follows the arguments used in [16] for the case $f(t)=t^{p}$.

Suppose by contradiction that $u$ does not vanish identically, then by the strong maximum principle and Hopf's lemma $u(t)>0$ if $t>0$ with $u(0)=0$, $u^{\prime}(0)>0, u^{\prime \prime}(t)=-f(u(t))<0$ for any $t>0$. Then the hypotheses imply that there exists $t_{0}>0$ such that $u^{\prime}\left(t_{0}\right)<0$.

In fact if this is not true then for any $t_{1}>0$ and $t \geq t_{1}, u(t) \geq a=u\left(t_{1}\right)>$ 0 , and since $f$ is continuous, positive in $(0, \infty)$ and $\liminf _{t \rightarrow \infty} f(t)>0$, there exists $m>0$ such that $f(s) \geq m \forall s \geq a$, in particular $u^{\prime \prime}(t)=-f(u(t)) \leq$ $-m<0$ for all $t \geq t_{1}$. By Taylor's theorem this implies that $u(t) \leq$ $u\left(t_{1}\right)+u^{\prime}\left(t_{1}\right)\left(t-t_{1}\right)-\frac{1}{2} m\left(t-t_{1}\right)^{2}$ so that $\lim _{t \rightarrow \infty} u(t)=-\infty$, a contradiction.

On the other hand if $u^{\prime}\left(t_{0}\right)<0$, since $u^{\prime \prime} \leq 0$ we get again by Taylor's formula that $u(t) \leq u\left(t_{0}\right)+u^{\prime}\left(t_{0}\right)\left(t-t_{0}\right) \rightarrow-\infty$ as $t \rightarrow \infty$.

\section{Nonexistence results for Problem (1.5)}

We begin by proving the following theorem which is a first generalization of the nonexistence result in [7] (where the nonlinearity $f(t)=t^{\frac{N+2}{N-2}}$ was studied and nonexistence in $D^{1,2}\left(\mathbb{R}_{+}^{N}\right)$ was proved) and will be needed in the subsequent proof of the main theorem (Theorem 1.1).

As in Section 1, let us put $A=\mathbb{R}_{+}^{N} \cup \Gamma_{1}$ and $W=\left\{\varphi \in C_{c}^{1}\left(\overline{\mathbb{R}_{+}^{N}}\right)\right.$ : $\operatorname{supp}(\varphi) \subseteq A\}$. Let $V$ be the completion of $W$ under the norm $\|\varphi\|=$ $\int_{\mathbb{R}_{+}^{N}}|\nabla \varphi|^{2}$. Then $V=\left\{u \in D^{1,2}\left(\mathbb{R}_{+}^{N}\right): u=0\right.$ on $\left.\Gamma_{0}\right\}$ where $D^{1,2}\left(\mathbb{R}_{+}^{N}\right)=$ $\left\{u \in L^{2^{*}}\left(\mathbb{R}_{+}^{N}\right):|\nabla u| \in L^{2}\left(\mathbb{R}_{+}^{N}\right)\right\}$.

Theorem 3.1 Suppose that $f(0) \geq 0$ and there exist $C, \alpha>0$ such that if $0 \leq a<b$ then

$$
\left|\frac{f(b)-f(a)}{b-a}\right| \leq C(a+b)^{\alpha}
$$

Then there are not nonzero weak solutions of (1.5) that belongs to $V \cap L^{\alpha \frac{N}{2}} \cap$ $C^{0}\left(\mathbb{R}_{+}^{N}\right)$ 
Remark 3.1 In the critical case, i.e. when $\alpha=2^{*}-2=\frac{4}{N-2}$, we have that $\alpha \frac{N}{2}=\frac{2 N}{N-2}=2^{*}$, and the hypothesis reduces to $u \in L^{2^{*}}$.

Proof of Theorem 3.1. We use the same notations as in the proof of Proposition 2.1 with the obvious modifications, e.g. $\Sigma_{\lambda}$ consists of the points $x$ belonging to $\mathbb{R}_{+}^{N}$ such that $x^{1}>\lambda$. We want to prove that the set $\Lambda$ of those $\lambda \in \mathbb{R}$ such that $u \leq u_{\mu}$ in $\Sigma_{\mu}$ for every $\mu \in(\lambda,+\infty)$ coincides in fact with $\mathbb{R}$, i.e. the solution $u$ is monotone decreasing in the $x^{1}$ - direction, which is impossible if $u$ belongs to some $L^{p}$ space, unless $u$ vanishes identically.

First of all the strong maximum principle holds, because $f(0) \geq 0$ and the hypotheses imply that $f$ is locally Lipschitz continuous, so that if $u$ does not vanish identically then $u$ is positive in $\mathbb{R}_{+}^{N}$.

Next to prove that $\lambda_{0}=\inf \Lambda=-\infty$ it suffices to prove that if $\lambda_{0}$ is finite then $u$ must coincide with reflection $u_{\lambda_{0}}$.

In fact this last possibility cannot hold due to the boundary conditions: if for a nontrivial solution $u$ and some $\lambda>0 u \equiv u_{\lambda}$ in $\Sigma_{\lambda}$, then $u$ would be a nontrivial solution of the Dirichlet BVP (1.6) and would be $C^{1}$ up to the boundary with $\frac{\partial u}{\partial x^{N}}>0$ on the boundary hyperplane by the Hopf's Lemma, contradicting the boundary condition on $\Gamma_{1}$ (recall that $f(0) \geq 0$ and $f$ is locally Lipschitz continuous by the hypotheses).

So the proof consists in the two usual steps in the moving plane method, and it will be completely analogous to that of Proposition 2.1 once we prove the analogue of Lemma 2.1, namely the following

Claim. There exists $C_{1}>0$, depending on the constant $C$ in (3.1) and on the dimension $N$, such that for each $\lambda \in \mathbb{R}$ the following holds

$$
\int_{\Sigma_{\lambda}}\left|\nabla\left(u-u_{\lambda}\right)^{+}\right|^{2} d x \leq C_{1}\left(\int_{A_{\lambda}} u^{\alpha \frac{N}{2}} d x\right)^{\frac{2}{N}} \int_{\Sigma_{\lambda}}\left|\nabla\left(u-u_{\lambda}\right)^{+}\right|^{2} d x
$$

where now $A_{\lambda}=\left\{x \in \Sigma_{\lambda}: u(x) \geq u_{\lambda}(x)\right\}$.

To prove the Claim we observe that if $\lambda \in \mathbb{R}$ and $u \in V$ weakly solves (1.5), the reflected function $u_{\lambda}$ weakly solves the problem

$$
\begin{cases}-\Delta u_{\lambda}=f\left(u_{\lambda}\right) & \text { in } \Sigma_{\lambda} \\ u_{\lambda}=0 & \text { on } \Sigma_{\lambda} \cap R_{\lambda}\left(\Gamma_{0}\right) \\ \frac{\partial u_{\lambda}}{\partial x^{N}}=0 & \text { on } \Sigma_{\lambda} \cap R_{\lambda}\left(\Gamma_{1}\right)\end{cases}
$$

So a test functions for this problem must vanish on $\Sigma_{\lambda} \cap R_{\lambda}\left(\Gamma_{0}\right)$, if nonempty, while a test function for the problem (1.5) must vanish on $\Sigma_{\lambda} \cap \Gamma_{0}$. In particular for any $\lambda \in \mathbb{R}$ the function $\left(u-u_{\lambda}\right)^{+}$vanishes in those sets. 
Since $u \in V$ there exists a sequence $\varphi_{j} \subset W$ such that

$$
\begin{cases}\varphi_{j} \rightarrow u & \text { in } L^{2^{*}}\left(\mathbb{R}_{+}^{N}\right) \\ \nabla \varphi_{j} \rightarrow \nabla u & \text { in } L^{2}\left(\mathbb{R}_{+}^{N}\right)\end{cases}
$$

and up to a subsequence $\varphi_{j} \rightarrow u$ and $\nabla \varphi_{j} \rightarrow \nabla u$ almost everywhere in $\mathbb{R}_{+}^{N}$. Let $\psi_{j}=f_{1 / j}\left(\varphi_{j}-\left(\varphi_{j}\right)_{\lambda}\right)$ where $f_{\varepsilon} \in C^{1}(\mathbb{R})$ is e.g.

$$
f_{\varepsilon}(t)= \begin{cases}\left(t^{2}+\varepsilon^{2}\right)^{\frac{1}{2}}-\varepsilon & \text { if }>0 \\ 0 & \text { if } t \leq 0\end{cases}
$$

Then $\psi_{j}$ is a good test function both for $u$ and $u_{\lambda}$ in $\Sigma_{\lambda}$ as it is immediate to see, for every $\lambda \in \mathbb{R}$. Testing the equations for $u$ and $u_{\lambda}$ in $\Sigma_{\lambda}$ with $\varphi$, subtracting the equations and passing to the limit as $j \rightarrow \infty$ we get, using the hypothesis, that

$$
\begin{aligned}
& \int_{\Sigma_{\lambda}}\left|\nabla\left(u-u_{\lambda}\right)^{+}\right|^{2} d x=\int_{\Sigma_{\lambda}}\left[f(u)-f\left(u_{\lambda}\right)\right]\left(u-u_{\lambda}\right)^{+} d x \\
& \quad \leq C \int_{A_{\lambda}}\left(u_{\lambda}+u\right)^{\alpha}\left(u-u_{\lambda}\right)\left(u-u_{\lambda}\right)^{+} d x \leq C^{\prime} \int_{A_{\lambda}} u^{\alpha}\left[\left(u-u_{\lambda}\right)^{+}\right]^{2} d x
\end{aligned}
$$

Using Holder and Sobolev inequalities as in Section 2 we get

$$
\begin{aligned}
\int_{\Sigma_{\lambda}}\left|\nabla\left(u-u_{\lambda}\right)^{+}\right|^{2} d x \leq C^{\prime} & \left(\int_{A_{\lambda}} u^{\alpha \frac{N}{2}} d x\right)^{\frac{2}{N}}\left(\int_{\Sigma_{\lambda}}\left[\left(u-u_{\lambda}\right)^{+}\right]^{2^{*}} d x\right)^{\frac{2}{2^{*}}} \\
& \leq C_{1}\left(\int_{A_{\lambda}} u^{\alpha \frac{N}{2}} d x\right)^{\frac{2}{N}} \int_{\Sigma_{\lambda}}\left|\nabla\left(u-u_{\lambda}\right)^{+}\right|^{2} d x
\end{aligned}
$$

Proceeding as in the proof of Proposition 2.1 it is easy to conclude.

Let us finally come to the proof of the main result.

Proof of Theorem 1.1. The proof of the fact that $u$ depends only on $x^{1}$ and $x^{N}$ is similar to the proof of Theorem 1.3 and consists in proving the symmetry of the Kelvin transforms of $u$ centered at any point $P$ with $P^{1}=P^{N}=0$ and with respect to any direction orthogonal to the $x^{1}$ and $x^{N}$ - directions.

To this end we observe that the Kelvin transforms $v$ of the solution $u$, as well as their reflections with respect to the directions in the subspace spanned by $e_{2}, \ldots, e_{N-1}$, satisfy the same mixed BVP as the solution $u$. Therefore it suffices to change the allowed test function to follow the preceding proofs closely in the case where $g$ is not constant on the values of $u$. In the case when $f(t)=l t^{\frac{N+2}{N-2}}$, the case $\lambda_{0}>0$ cannot occur, for the original solution $u$ would be regular at infinity and Theorem 3.1 shows that there are not such solutions. 
Since the center of the Kelvin transform is an arbitrary point $P$ with $P^{1}=P^{N}=0$, and $u$ is symmetric with respect to the hyperplanes through $P$ and orthogonal to any direction orthogonal to the $x^{1}$ and $x^{N}$ - directions, it follows that $u$ does not depend on $x^{2}, \ldots x^{N-1}$.

For what concerns the monotonicity in the $x_{1}$-direction we proceed at first in the same way, by considering the Kelvin transform $v$ of $u$ centered at the origin, which satisfies the same boundary value problem, and proving that the inequality $v<v_{\lambda}$ holds true in $\Sigma_{\lambda}$ provided $\lambda>0$ (the only difference is that now we cannot start moving the hyperplanes in the opposite direction). By continuity we get that $v \leq v_{0}$ in $\Sigma_{0}=\left\{x \in \mathbb{R}_{+}^{N}: x_{1}>0\right\}$. Recalling the definition of $v(x)=\frac{1}{|x|^{N-2}} u\left(\frac{x}{|x|^{2}}\right)$ and observing that $\left|x_{\lambda}\right|=|x|$ for $\lambda=0$, we get that $u \leq u_{0}$ in $\Sigma_{0}$.

The same trick works when considering the Kelvin transform $v^{\mu}(y)=$ $v_{P_{\mu}}(y)=\frac{1}{|y|^{N-2}} u\left(P_{\mu}+\frac{y}{|y|^{2}}\right)$ centered at a point $P=P_{\mu}$ with $P^{2}=\cdots=P^{N}=$ $0, P^{1}=\mu \geq 0$. It satisfies a Dirichlet condition in the part of the boundary consisting of points $y$ with $y^{N}=0, y^{1}>0$ and this is enough to prove as before that for any $\lambda>0$ the inequality $v^{\mu} \leq v_{\lambda}^{\mu}$ holds in $\Sigma_{\lambda}$. Since $\lambda>0$ is arbitrary we get $v^{\mu} \leq v_{0}^{\mu}$ in $\Sigma_{0}$, i.e. $v^{\mu}\left(y^{1}, y^{\prime}\right) \leq v_{0}^{\mu}(y)=v^{\mu}\left(-y^{1}, y^{\prime}\right)$ for every $y=\left(y^{1}, y^{\prime}\right)$ with $y^{1}>0$. In terms of $u$ this means that the inequality $u \leq u_{\mu}$ holds in $\Sigma_{\mu}$ for each $\mu \geq 0$, and in particular $u$ is nonincreasing in the $x^{1}$-direction in the part of the half space where $x^{1}>0$.

The proof of the monotonicity in the $x^{1}$-direction in the whole half space instead requires new ideas, since the preceeding technique does not work. In fact we would like to prove that the relation $u \leq u_{\mu}$ holds in $\Sigma_{\mu}$ also when $\mu<0$, but here there is a problem in using the Kelvin transform $v^{\mu}(y)=\frac{1}{|y|^{N-2}} u\left(P+\frac{y}{|y|^{2}}\right)$ centered at $P=P_{\mu}=(\mu, \ldots, 0)$ : it is impossible to start the moving plane method for this transform from $\lambda=+\infty$ since if $\lambda$ is big there are points where the Neumann condition holds, and therefore the solution is positive, which are reflected in points where the function vanishes. In terms of test function, for $\lambda$ big the function $\left(v-v_{\lambda}\right)^{+}$is not allowed as a test function for the problem satisfied by the reflected function $v_{\lambda}$, since it does not vanish in points where the Dirichlet condition for $v_{\lambda}$ holds.

Nevertheless the main estimate used in all the preceeding theorems holds if $\lambda$ is close to zero, so the idea is to continue the inequality $v^{\mu}(y)<v^{\mu}\left(y_{\lambda}\right)$ for every $\lambda>0$ fixed, moving $\mu$ from $\mu=0$, where the strict inequality is true (since $\lambda>0$ ), down to $\mu=\frac{-1}{2 \lambda}$.

Let us now make precise these considerations.

If $\mu \leq 0$ let $v^{\mu}$ be the Kelvin transform of the solution centered at the point $P_{\mu}=(\mu, 0, \ldots, 0)$, i.e. $v^{\mu}(y)=\frac{1}{|y|^{N-2}} u\left(P_{\mu}+\frac{y}{|y|^{2}}\right)$. It satisfies a Dirichlet boundary condition in points $y$ with $y^{N}=0,0<y^{1}<-1 / \mu\left(0<y^{1}<\infty\right.$ if $\mu=0$ ) and a Neumann condition in the remaining part of the boundary. 
If $0<\lambda<\frac{-1}{2 \mu}$ then $v^{\mu}$, and therefore also $\left(v^{\mu}-v_{\lambda}^{\mu}\right)^{+}$, vanishes not only where the Dirichlet condition holds for $v=v^{\mu}$, but also where the Dirichlet condition holds for the reflected function $v_{\lambda}^{\mu}$. Therefore we can take, as in Lemma 2.1, the function $\varphi=\varphi_{\varepsilon}=\eta_{\varepsilon}^{2}\left(v^{\mu}-v_{\lambda}^{\mu}\right)^{+}$as a test function in the problems satisfied by $v^{\mu}$ and $v_{\lambda}^{\mu}$. Proceeding exactly as in the lemma we get the main estimate, namely

$$
\int_{\Sigma_{\lambda}}\left|\nabla\left(v^{\mu}-v_{\lambda}^{\mu}\right)^{+}\right|^{2} \leq C_{\lambda}\left(\int_{A_{\lambda}^{\mu}} \frac{1}{|y|^{2 N}}\right)^{2 / N}\left(\int_{\Sigma_{\lambda}}\left|\nabla\left(v^{\mu}-v_{\lambda}^{\mu}\right)^{+}\right|^{2}\right)
$$

where $C_{\lambda}$ depends on $N, \lambda$, is nonincreasing in $\lambda$ and $A_{\lambda}^{\mu}=\left\{y \in \Sigma_{\lambda} \backslash P_{\lambda}\right.$ : $\left.g\left(|y|^{N-2} v^{\mu}(y)\right)>0, v^{\mu}(y) \geq v_{\lambda}^{\mu}(y)\right\}$.

If we now fix $\lambda>0$, then the previous estimate holds for every $\mu \in$ $\left(\frac{-1}{2 \lambda}, 0\right)$. Moreover the function $\frac{1}{|y|^{2 N}} \chi_{A_{\lambda}^{\mu}}$, where $\chi_{S}$ stands for the characteristic function of the set $S$, converges pointwisely to zero as $\mu \rightarrow 0$ in $\mathbb{R}^{N} \backslash\left(T_{\lambda} \cup\left\{P_{\lambda}\right\}\right)$ (recall that $P_{\lambda}=(2 \lambda, 0, \ldots, 0)$ is the reflected point of the origin, which is the singular point of every transform $\left.v^{\mu}\right)$.

Proceeding exactly as in the proof of Proposition 2.1 we deduce that the inequality $v^{\mu} \leq v_{\lambda}^{\mu}$ holds in $\Sigma_{\lambda}$ for $\mu<0$ and close to 0 , and the same arguments permit to continue downto $\mu=\frac{-1}{2 \lambda}$.

So we get that for every $\lambda>0$ and every $\mu \geq \frac{-1}{2 \lambda}$ the inequality $v^{\mu} \leq v_{\lambda}^{\mu}$ holds in $\Sigma_{\lambda}$. Put in another form for each fixed $\mu<0$ the inequality holds for every $\lambda$ with $0<\lambda<\frac{-1}{2 \mu}$. Letting $\lambda \rightarrow 0$ we get that $v^{\mu} \leq v_{0}^{\mu}$ in $\Sigma_{0}$, i.e. $v^{\mu}\left(y^{1}, y^{\prime}\right) \leq v_{0}^{\mu}(y)=v\left(-y^{1}, y^{\prime}\right)$ for every $y=\left(y^{1}, y^{\prime}\right)$ with $y^{1}>0$. This implies as before that the inequality $u \leq u_{\mu}$ holds in $\Sigma_{\mu}$, and since $\mu<0$ is arbitrary we get that $u$ is nonincreasing in the $x^{1}$ - direction in the whole half space.

Finally suppose that $u$ is bounded and $f$ satisfies iii). Since $u$ depends only on $x^{1}$ and $x^{N}$ and it is decreasing in the $x^{1}$-direction, for every $t \geq 0$ there exists the limit $z(t)=\lim _{x^{1} \rightarrow-\infty} u\left(x^{1}, t\right)$ and it is easy to see that $z$ satisfies the same equation in $\mathbb{R}_{+}$(see e.g. [12]), with a Neumann condition in 0 . Then proceeding as in the final part of Theorem 1.3 we conclude easily that $z \equiv 0$ and therefore, by monotonicity, $u \equiv 0$.

Remark 3.2 Suppose that ( $f(0) \geq 0$, which is a consequence of the hypothesis (i) and that ) $f$ is the sum of a Lipschitz function and a nondecreasing function. As in Theorem 3.1 if $u$ is a nontrivial solution the equality $u \equiv u_{\lambda}$ cannot hold in $\Sigma_{\lambda}$ for any $\lambda \in \mathbb{R}$ because of the boundary conditions. Then by the strong comparison principle and the Hopf's Lemma we get $u<u_{\lambda}$ in $\Sigma_{\lambda}$ and $\left(u-u_{\lambda}\right)_{x^{1}}=2 u_{x^{1}}<0$ on $T_{\lambda} \cap \mathbb{R}_{+}^{N}$. Since $\lambda \geq 0$ is arbitrary we get that $u$ depends only on $x^{1}$ and $x^{N}$ and $u_{x^{1}}(x)<0$ for every $x \in \mathbb{R}_{+}^{N}$. 


\section{References}

[1] Almeida, L., Damascelli, L., Ge, Y.: A few symmetry results for nonlinear elliptic PDE on noncompact manifolds. Ann. Inst. H. Poincaré Anal. Non Linéaire 19 (2002), no. 3, 313-342.

[2] Bianchi, G.: Non-existence of positive solutions to semilinear elliptic equations on $\mathbb{R}^{N}$ or $\mathbb{R}_{+}^{N}$ through the method of moving planes. Comm. Partial Differential Equations 22 (1997), no. 9 10, 1671-1690.

[3] Berestycki, H., Caffarelli, L. A. and Nirenberg, L.: Symmetry for elliptic equations in a half space. In Boundary value problems for partial differential equations and applications, 27-42. RMA Res. Notes Appl. Math. 29. Masson, Paris 1993.

[4] Berestycki, H., Caffarelli, L. A. And Nirenberg, L.: Inequalities for second order elliptic equations with applications to unbounded domains I. Duke Math J. 81 (1996), 467-494.

[5] Berestycki, H., Caffarelli, L. A. and Nirenberg, L.: Monotonicity for elliptic equations in unbounded Lipshitz domains. Comm. Pure Appl. Math. 50 (1997), 1089-1111.

[6] Berestycki, H., Caffarelli, L. A. and Nirenberg, L.: Further qualitative properties for elliptic equations in unbounded domains. Ann. Scuola Norm. Sup. Pisa Cl. Sci. 25 (1997), 69-94.

[7] Berestycki, H., Grossi, M. and Pacella, F.: A nonexistence theorem for an equation with critical Sobolev exponent in the half space. Manuscripta Math. 77 (1992), 265-281.

[8] Caffarelli, L. A., Gidas, B. And Spruck, J.: Asymptotic symmetry and local behavior of semilinear elliptic equations with critical Sobolev growth. Comm. Pure Appl. Math. 42 (1989), 271-297.

[9] Chen, C. C. And Lin, C. S.: Local behavior of singular positive solutions of semilinear elliptic equations with sobolev exponent. Duke Math. J. 78 (1995), no. 2, 315-334.

[10] Chen, W. And Li, C.: Classification of solutions of some nonlinear elliptic equations. Duke Math. J. 63 (1991), no. 3, 615-622.

[11] Colorado Heras, E. And Peral Alonso, I.: Semilinear elliptic problems with mixed Dirchlet-Neumann boundary conditions. J. Funct. Anal. 199 (2003), no. 2, 468-507.

[12] Dancer, E. N.: Some notes on the method of moving planes. Bull. Austral. Math. Soc. 46 (1992), 425-434.

[13] Damascelli, L., Ramaswamy, M.: Symmetry of $C^{1}$ solutions of $p$-Laplace equations in $R^{N}$. Adv. Nonlinear Stud. 1 (2001), no. 1, 40-64.

[14] Gidas, B., Ni, W. M. and Nirenberg, L.: Symmetry of positive solutions of nonlinear elliptic equations in $\mathbb{R}^{N}$. In Mathematical analysis and applications, Part A, 369-402. Adv. in Math. Suppl. Stud. 7a. Academic Press, New York-London, 1981. 
[15] Gidas, B., Spruck, J.: Global and local behavior of positive solutions of nonlinear elliptic equations. Comm. Pure Appl. Math. 34 (1981), 525-598.

[16] Gidas, B., Spruck, J.: A Priori Bounds for positive solutions of nonlinear elliptic equations. Comm. Partial Differential Equations 6 (1981), 883-901.

[17] Lou, Y., ZHu, M.: Classification of nonnegative solutions to some elliptic problems. Differential Integral Equations 12 (1999), no. 4, 601-612.

[18] Pucci, P., Serrin, J. And Zou, H.: A strong maximum principle for quasilinear elliptic equations. J. Math. Pures Appl. (9) 78 (1999), 769-789.

[19] Protter, M. H., Weinberger, H. F.: Maximum Principle in Differential Equations. Prentice-Hall, Inc., Englewood Cliffs, N.J., 1967.

[20] Terracini, S.: Symmetry properties of positive solutions to some elliptic equations with nonlinear boundary conditions. Differential Integral Equations 8 (1995), 1911-1922.

[21] Terracini, S.: On positive entire solutions to a class of equations with a singular coefficient and critical exponent. Adv. Differential Equations 1 (1996), no. 2, 241-264.

[22] VÁzquez, J. L.: A Strong maximum principle for some quasilinear elliptic equations. Appl. Math. Optim. 12 (1984), 191-202.

Recibido: 12 de febrero de 2002

Lucio Damascelli

Dipartimento di Matematica Università di Roma "Tor Vergata"

Via della Ricerca Scientifica 00133 Roma, Italy

damascel@mat.uniroma2.it

Francesca Gladiali

Dipartimento di Matematica Università di Roma "La Sapienza"

P.le A. Moro 2

00185 Roma, Italy

gladiali@mat.uniroma1.it

Supported by MURST, Project "Metodi Variazionali ed Equazioni Differenziali Non Lineari". 